\title{
SUBARACHNOID HAEMORRHAGE AS A CAUSE OF DEATH : A REVIEW OF FORENSIC AUTOPSIES CONDUCTED IN EDINBURGH
}

\author{
P.A.S. Edirisinghe \\ Senior Lecturer, Department of Forensic Medicine, Faculty of Medicine, \\ University of Kelaniya, Sri Lanka.
}

\section{INTRODUCTION}

Subarachnoid haemorrhage (SAH) as a cause of death in autopsies where death occurred before reaching medical attention has not changed over the years ${ }^{1}$. Although majority of the cases are spontaneous in nature due to natural causes, occasional SAH due to trauma is encountered in forensic practice. Subarachnoid haemorrhage (SAH) comprises $1 \%$ to $7 \%$ of all strokes, therefore it is important in morbidity and mortality, especially in the elderly. Although the diagnosis of SAH has advanced due to invent of imaging technology and campaign on preventive measures, the familial preponderance suggesting a genetic influence associated with harmful lifestyles has attributed to sudden deaths ${ }^{2,3}$. Macroscopic diagnosis of SAH at the autopsy is easy, but finding the underlying cause is at times difficult and time consuming. It is important to seek an easier and practical method in the investigation and diagnosis of underlying causes of SAH at the autopsy. Also it is pertinent to educate the relatives and the public about this condition.
Past medical history was obtained from the police notes provided by the GP. Immediate complaints were divided into three main groups according to the available data. ñComplaint of headacheò and ñcollapseò was taken when witnesses are available to give information while the category ófound deadôwas taken when no witness were available to comment on the period prior to death.

\section{RESULTS}

Sixty seven (67) (0.8\%) cases were diagnosed as $\mathrm{SAH}$ as the cause of death out of 8,264 post mortem examinations done during this period. There was a slight female preponderance in the sample where 37 were females while 30 were males. The majority was distributed in their fifth or sixth decade of life. The medium age of the sample was 59.3 years, the youngest being 19 years old while the oldest being 99 years old.

\begin{tabular}{|l|l|l|l|l|l|l|l|l|l|l|l|l|l|l|}
\hline & $<20$ & $20-$ & $25-$ & $30-$ & $35-$ & $40-$ & $45-$ & $50-$ & $55-$ & 60 & 65 & 70 & 75 & $80+$ \\
\hline Male & & 2 & 2 & 2 & 1 & 1 & 5 & 5 & 1 & 2 & 3 & 3 & 2 & 1 \\
\hline Female & & & 1 & & & 2 & 2 & 3 & 11 & 1 & & 7 & 3 & 7 \\
\hline
\end{tabular}

\section{METHOD}

All forensic cases at Edinburgh University, pathology division, in which death was certified as Subarachnoid Haemorrhage (SAH) as 1a or $1 \mathrm{~b}$ in the death certificate between 2000-2005 were reviewed. All these cases had been referred by Lothian and Borders Procurator Fiscal. They were autopsied and investigated at the Section of Forensic Medicine, division of Pathology, University of Edinburgh. Data were collected using a proforma, by reviewing the post-mortem examination reports and the case notes provided by the police. Descriptive analysis was done with a pre-tested protocol.
The cause of death (1a) was given as Acute SAH or Spontaneous SAH in majority of the cases (87\%) while the remaining varied between acute hydrocephalus, increased intracranial pressure, hypostatic bronchopneumonia, subdural hamemorrghage associated with SAH. There was only one case of traumatic subarachnoid haemorrhage in a young male due to blunt force trauma to the neck. There were 5 cases of SAH associated with trauma due to falls.

Out of 67 cases $51(76 \%)$ cases had óupture of cerebral artery aneurysmôas the underlying cause for spontaneous or acute subarachnoid haemorrhage as $1 \mathrm{~b}$ in the death certificate. 15 $(22 \%)$ of them had óupture of cerebral berry aneurysmô while one case has the diagnosis of rupture of cerebral atheromatic aneurysm. Three 
cases (4.5\%) had the diagnosis of rupture of cerebral artery atheroma as the underlying cause for acute/spontaneous SAH. All three cases were above 70 years and had generalized atheroma as an associated disease in the death certificate.

\begin{tabular}{|l|c|}
\hline Cause for the SAH & $\begin{array}{l}\text { Number of deaths } \\
\text { in the study }\end{array}$ \\
\hline $\begin{array}{l}\text { SAH due to aneurismal } \\
\text { rupture }\end{array}$ & 51 \\
\hline $\begin{array}{l}\text { SAH associated with } \\
\text { trauma }\end{array}$ & 5 \\
\hline Traumatic SAH & 1 \\
\hline $\begin{array}{l}\text { SAH due to other } \\
\text { causes/no cause }\end{array}$ & 5 \\
\hline \multicolumn{2}{|c|}{ Total } \\
\hline
\end{tabular}

$15(22 \%)$ cases had hypertension as an associated cause of death in death certificate and only 9 of them were on medication. 9 out of 67 had a diagnosis of generalized atheroma / coronary artery disease as an associated cause of death while 4 cases only had an associated diagnosis of fatty liver/cirrhosis of liver, where there was a history of high intake of alcohol.

21(40\%) had a past medical history of hypertension, ischemic heart disease or stroke while only two had a past history of SAH with aneurismal clipping. 26 had documentary evidence of a complaint of headache/ and or collapse while 33 were found dead beyond the state of resuscitation. Although, rupture of aneurysm causing sudden death in young while having sex/intercourse is mentioned in forensic texts, only 2 males in our series (53 and 36 years) had a similar history. Since use of prescribed drugs or self-medication for minor ailments is common in any society, 33 (49\%) had evidence of medication at the time of the death. One person had a history of drug abuse.

19 cases recoded presence un-ruptured aneurysms varying from $2 \mathrm{~mm}$ to $10 \mathrm{~mm}$. The majority of ruptured and un-ruptured aneurysms were located in the anterior part of the circle of Willis. Multiple aneurysms were recorded in 12 cases. Most of SAH were basal (37); nonetheless 11 cases had an associated intra ventricular haemorrhage. Toxicological examination was done on 8 cases, which was associated with trauma where alcohol was found in 3 cases, benzodiazepine in one and Ibuprofen in one case. All trauma associated SAH had a history of a fall. Two of them had contusions in the scalp and one had a fracture in the occipital area of the skull, while another had a dislocation of the shoulder. Histology was done in 5 cases, which were associated with trauma.

\section{DISCUSSION}

According to ICD 10 (International Classification of Diseases) SAH is classified as number 160. Although incidence of SAH has been estimated for many years at 10 to 15 per 100000 person-years, most recent studies show lower figures such as 6 to 8 per 100000 person-years ${ }^{4}$. However, Pobereskin report that the incidence rates are higher than those previously reported in the United Kingdom and the age standardized incidence rate for females is 11.9 , while the rate for males is $7.4^{5}$. Scotland recorded 93 male deaths and 163 female deaths due to SAH in the year 2000 for a population of just over 5 million ${ }^{6}$.

A death certificate has several purposes. It gives the necessary information as to the cause of death of an individual, which is in turn helps to fulfill legal rights of the individual, his family and the country he lives. Statistics on death also helps a government to plan health polices of a country. However, government statistics, which are categorized according to the ICD 10, does not divide $\mathrm{SAH}$ as that of acute or spontaneous. In our study most of the death certificates i.e. $87 \%$ carried this descriptive data. Although, it is important to the relative to understand the suddenness and the nature of the illness itô relevance as a standard document is a matter for debate. The meaning of the word ácuteôaccording to oxford dictionary in medical context is a disease coming sharply to a crisis, whereas spontaneous means acting or occurring without external cause, which describe the development of the illness. Therefore, when using this word in the death certificate the pathologist should be cautious because this can lead to many misunderstanding and misinterpretations regarding the peri-mortem period.

Earlier autopsy studies on SAH from Texas, U.S.A. (autopsies carried out during 1977-1997) states that SAH due to ruptured cerebral artery aneurysms accounted for $1.5 \%$ of natural deaths and $0.4 \%$ of all deaths during this period ${ }^{7}$. However the our study showed $0.6 \%$ cases of all deaths investigated during this period was due to rupture of a cerebral aneurysm. The high rate encountered in Edinburgh may be due to the poor health record for vascular disease in Scotland ${ }^{8,9}$. The incidence of intracranial aneurysms is unknown but autopsy studies reveal that approximately $4-5 \%$ of sudden natural deaths have 
a cerebral aneurysm ${ }^{10}$. However, more than $50 \%$ of aneurysms identified at post-mortem examinations are asymptomatic and previously unrecognized $^{10}$. In a meta-analysis of relevant studies, the pooled incidence rate of SAH due to ruptured aneurysms is 10.5 per 100000 person years ${ }^{4}$.

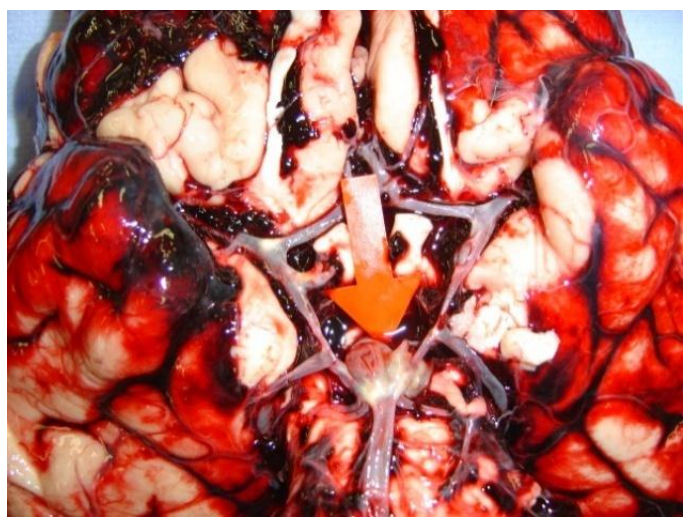

Approximately $85 \%$ of all spontaneous haemorrhages into the subarachnoid space arise from rupture of saccular aneurysms at the base of the brain ${ }^{11}$. Other causes of SAH are ruptured arteriorvenous malformations, intracranial artery dissections, mycotic aneurysms, trauma, bleeding disorders in patients whom no aneurysm is discovered on initial angiogram ${ }^{12,13}$. Saccular aneurysms that arise at arterial bifurcations in the subarachnoid space are also known as berry aneurysms. 15 cases in our study had the underlying cause as a rupture of a berry aneurysm while 51 cases had the underlying cause as rupture of a cerebral artery aneurysm. It is unknown why some persons develop aneurysms at arterial bifurcations and others do not.

The once popular notion of a congenital defect in the muscle layer of the wall (tunica media) being a weak spot through which the inner layers of the arterial wall would bulge has been largely dispelled by a number of contradictory observations $^{13}, 14$. With this background we suggest unless a careful macroscopic and histological diagnosis is made to identify the type of aneurysm (saccular, fusiform, mycotic or tramatic) it is better to have the phrase óupture of intracranial aneurysmô in the death certificate to avoid a medical controversy.

Two hypotheses have been put forward to explain the development of intracranial aneurysms ${ }^{15}$. One theory implies acquired factors such as mural atrophy due to hemodynamic disturbances associated with hypertension; this theory holds that the berry/saccular aneurysm is an acquired lesion arising from a weakness in the arterial wall associated with a high intraluminal pressure. Another theory cites congenital defects of the arterial wall similar to those seen in hereditary connective tissue disorders such as Marfanôs syndrome, Ehlers-Danlos syndrome, and pseudoxanthoma elasticum. Associated congenital abnormalities such as aortic coarctation, polycystic disease of the kidneys, and arteriovenous malformations, support this theory.

Although several risk factors are known to predispose a person for a $\mathrm{SAH}$, cigarette smoking, hypertension and alcohol abuse are the most important ${ }^{16,17}$. In keeping with common trend $40 \%$ of our study had documentary evidence of a past history of hypertension and $86 \%$ of them were confirmed at the autopsy. However autopsy studies on rupture of cerebral aneurysm from Belgrade and Texas showed higher rates of a history of hypertension $(65-75 \%)^{18,7}$. This indicates the need of screening for hypertension to be more strengthened at the primary care level in Scotland. We did not study about the smoking and alcohol in this group since the information provided by the police about those habits were not mentioned in every case. Another interesting factor in this study was half of the people who died of SAH were taking some medicinal drug at the time of death. This indicates that although majority of diagnosed hypertensives were on medication it was not controlled adequately.

However, SAH tend to occur in ambulatory patients and following physical exertion; therefore sudden rise in blood pressure may play a part in rupture of aneurysm rather than high blood pressure $^{14}$. Prescribed and non-prescribed medication of non-steroidal anti-inflammatory drugs and anticoagulants used by elderly may also contribute to a SAH. Although the development of imaging technology and neuro-surgery in past two decades was rapid, we had encountered only two cases of previously diagnosed $\mathrm{SAH}$, and aneurysmal clipping 2-3 years ago. The main reason for not encountering this group in forensic autopsy population is that these deaths may have been investigated as a pathological post-mortems rather than an investigation of sudden death.

The sudden nature of this condition is again highlighted in this study. 26 had documentary evidenced of development of headache and collapse prior to death, while 33 were found dead which adds to $85 \%$ from the total who die before reaching hospital or medical attention. The combined overall risk of sudden death before medical attention according eighteen populationbased studies between 1965 and 2001 to was 
$12.4 \%$ for aneurysmal SAH (95\% confidence interval, $11-14 \%$ ) and $44.7 \%$ for posterior circulation aneurysms. ${ }^{19}$ However, our autopsy study showed the majority of the aneurysms were located in the anterior part of the circle of Willis that is similar to other autopsy studies ${ }^{7,18}$.

Most intracranial aneurysms increase in size and/or rupture with time. Aneurysms greater than $10 \mathrm{~mm}$ in diameter have a roughly $50 \%$ risk of bleeding per year'. Ruptured aneurysms are usually larger than un-ruptured ones, and it is now known when aneurysms reach a critical size (7$10 \mathrm{~mm}$ ), the probability of hemorrhage increases ${ }^{20}$. Our autopsy study too showed that the presence of un-ruptured aneurysms could be varied in sizes. Multiple aneurysms were found in $17 \%$ of cases compared to $12 \%$ in Texas study. SAH can cause virtually instantaneous death even though the mechanism is obscure. The location of the ruptured aneurysm is significant in regard to survival time. Patients whose aneurysm is located on the internal carotid artery or on the posterior part of the circle of Willis have a greater chance of dying at the time of rupture (69\% to $79 \%)$ than those with an aneurysm on the middle cerebral, anterior cerebral, and anterior communicating arteries $(49 \% \text { to } 55 \%)^{19}$.

Exertion has been linked to aneurysmal rupture. However studies report that nearly half of the patients are engaged in non-strenuous activities such as resting or sleeping at the time of rupture while the others are engaged in stressful activities just before rupture (eg, heavy work, exercise, sexual intercourse) or have a heightened degree of emotional tension ${ }^{21}$. Our study revealed only two cases of such heightened degree of emotional tension where as almost 50\% (33) were found dead where there was no reliable evidence or records to comment about the immediate activity prior to death.

Mortality rate of SAH is proportional to the amount of blood released to the subarachnoid space. Those who have a haemorrhage of $15 \mathrm{~cm}^{3}$ or less have a better prognosis ${ }^{22}$. However, a significant proportion of SAH deaths have a massive intraventicular haemorrhage usually from ruptured anterior communicating artery aneurysm or intra-cerebral bleeding form middle cerebral aneurysms ${ }^{23}$. Rupture of an aneurysm usually occurs at the fundus and rarely at the sides or the neck of the aneurysm. If the aneurysm is in the anterior half of the circle of Willis, SAH will be basal, extending into the inter-peduncular fossa and over the convexities of the frontal and parietal lobes. Although exact cause of death when blood is confined to the subarachnoid space is unclear, death may be due to a sudden rush of blood at arterial pressure into the subarachnoid space leading to meningeal irritation with cortical oedema. If the aneurysm is embedded in the cortex, there may be intra-cerebral or intraventricular hemorrhage and obstruction of the aqueduct by blood, with subsequent hydrocephalus. Accumulation of a large hematoma will produce increased mass effect, possibly with secondary herniations and ischemia ${ }^{24}$. In this study most of the SAH were basal while there was a considerable amount of intraventicular haemorrhages indicating the lethality of the episode.

Another form of severe basal SAH is known to occur with laceration of the vertebral/ basilar artery due to trauma. Hyperextension of the head can cause injury to the vessels at the base of the brain. These lacerations are believed to be caused by stretching of the vessels due to the abrupt movement of the head and rotational acceleration of the brain within the cranium. They usually occur in the intracranial portions of the vessels, producing a basal SAH. Most of these deaths have evidence of trauma to the neck or side of the face or $\mathrm{jaw}^{25}$. In this study only one case had the diagnosis of a traumatic SAH with a punch to the side of the neck while 5 cases had an association with trauma. Hence, the importance of doing a careful neck dissection is highlighted in suspected cases. In trauma associated SAH, it is essential to have a careful dissection of the Circle of Willis to avoid missing an underlying aneurysm.

There are two choices a pathologist can have for examination of the brain at the autopsy. One method is to dissect the circle of Willis and its major branches free from the brain and identification of the aneurysm on exposed vessels. The other is to section the fixed brain coronally in $1 \mathrm{~cm}$ and examine macroscopically and microscopically. Whatever the choice is made, if results of prior angiography are available it greatly facilitate the location of the aneurysm. Although fixing the brain is time consuming, it is a good method if the ruptured aneurysm is very small.

In similar to other autopsy studies on SAH, this study also showed that the patterns and the prior diagnosis have not changed despised the improvement of the imaging technology and increase availability to access to medical information and advice. This indicates that awareness of this condition among the common man is not adequate. A significant proportion of this condition has a familial preponderance 
suggesting a genetic influence with harmful lifestyles; therefore it is our duty to learn lessons from the dead and to strengthen the preventive measures.

In the advent of non-invasive methods of imaging intracranial blood vessels has facilitated screening for intracranial aneurysms in people who are at risk. A strong risk factor for SAH from an aneurysm is a positive family history, defined as two or more first-degree relatives with $\mathrm{SAH}$ (relative risk $[R R]=6.6$ ). The greatest familial risk is associated with an affected sibling. Another strong risk factor is polycystic kidney disease $(\mathrm{RR}=4.4)$. People who have these risk factors are potential candidates for screening ${ }^{26}$. As forensic pathologists who investigate sudden deaths it is our duty to inform the family of the deceased about the possibility of screening particularly if there have been any symptoms and advice on hypertension control, smoking and alcohol through their own GP if we are to prevent another sudden death from $\mathrm{SAH}$.

\section{ACKNOWLEDGEMENT}

Professor A Busuttil, Forensic Medicine: Division of Pathology, University of Edinburgh for guidance.

\section{REFERENCE}

1. Schievink WI, Wijdicks EP, Piepgras DG, Whisnant JP. Sudden death from aneurismal subarachnoid haemorrhage. Neurology 1995;45(5):871-4.

2. Ruigrok YM, Buskens E, Rinkel GJ. Attributable risk of common and rare determinants of subarachnoid hemorrhage. Stroke. 2001; 32: 1173ï 1175.

3. Feigin VL, Lawes CM, Bennett DA, Anderson CS. Stroke epidemiology: a review of population-based studies of incidence, prevalence, and case-fatality in the late 20th century. Lancet Neurol. 2003; 2: $43 \ddot{i} 53$

4. Linn FH, Rinkel GJ, Algra A, van Gijn J. Incidence of subarachnoid haemorrhage: role of region, year and rate of computed tomography: a meta analysis. Stroke 1996:27:625-9

5. Pobereskin $\mathbf{L} \mathbf{H}$. Incidence and outcome of subarachnoid haemorrhage: a retrospective population based study. J Neurol Neurosurg \& Psych 2001;70:340-343

6. Annual report of the Register General for Scotland 2000. http://groscotland.gov.uk/statistics/library/annrep/index.html Web site consulted on 3.2.2006

7. Gonsoulin M, Barnard Jeffrey J, Prahlow J A., Death Resulting from Ruptured Cerebral Artery Aneurysm: 219 Cases. Ame J Forensic Medicine \& Pathology 2002;23(1):5-14.

8. Elford J, Phillips AN, Thomson AG, Shaper AG. Migration and geographic variations in ischaemic heart disease in Great Britain. Lancet 1989;1:343ї 346.

9. Syme P D., Byrne A W., Chen R, Devenny R, Forbes J F. Community-Based Stroke Incidence in a Scottish Population. Stroke 2005;36:1837

10. Vinas FC, Wilner HI, Brain, Aneurysm http://www.emedicine.com/consulted 27.1.2005

11. Van Gijn J, van Dongen KJ. Computed tomography in the diagnosis of subarachnoid haemorrhage and ruptured aneurysm. Clin Neurol Neurosurg 1980; 82: 11 ï 24.

12. Rinkel GJE, J. van Gijn, Wijdeks Subarachnoid haemorrhage without detectable aneurysm. A review of causes. Stroke 1993;24:1403-

13. J. van Gijn and G. J. E. Rinkel, Subarachnoid haemorrhage: diagnosis, causes and management. Brain 2001; 124: 249-278

14. Weller RO. Subarachnoid hemorrhage and myths about saccular aneurysm. J Clin Pathol 1995; 48: 1078-81 
15. Ostergaard JR: Risk factors in intracranial saccular aneurysms: aspects on the formation and rupture of aneurysm, and development of cerebral vasospasm. Acta Neurol Scand 1989; 80:81-98

16. Teunissen LL, Rinkel GJE, Algra A, van Gijn J. Risk factors for subarachnoid hemorrhage ï a systematic review. Stroke 1996; 27: $544 і ̈ 9$.

17. Feigin VL.; Rinke G J.E. 1, Lawes C M.M., Algra A, Bennett D A., J van Gijn, Anderson C S., Risk Factors for Subarachnoid Hemorrhage An Updated Systematic Review of Epidemiological Studies. Stroke 2005;36:2773

18. Nikolic S, Banjanin I, Stanojevic A. Subarachnoidal hemorrhage from saccular aneurysms as a cause of natural death. Srp Arh Celok Lek. 132(7-8):236-9.

19. Huang J,van Gelder, J M. The Probability of Sudden Death from Rupture of Intracranial Aneurysms: A Meta-analysis. Neurosurgery. 2002;51(5):1101-1107.

20. Juvela S, Porras M, Poussa K: Natural history of unruptured intracranial aneurysms: probability of and risk factors for aneurysm rupture. J Neurosurg, 2000; 93(3): 379-87
21. Mocco J, Komotar RJ, Lavine SD, Meyers PM, Connolly ES, Solomon RA. The natural history of unruptured intracranial aneurysms. Neurosurg Focus. 2004 15;17(5):E3.

22. Sacco RI, Wolf PA, Kannel WB, McNamara PM. Survival and recurrence following stroke. The Framingham Study. Stroke 1982;13:290-95

23. Inagawa $\mathrm{T}$, Hirano $\mathrm{A}$, Ruptured intracranial aneurysms: an autopsy study of 133 patients. Surg Neurol 1990;33:117-23

24. Stehbens WE: Aneurysms. Vascular Pathology. Chapman and Hall Medl Lond, 1995, pp 379-400

25. Kindelberger D, Gilmore K, Catanese CA, Armbrustmacher VW. Hyperextension and rotation of head causing internal carotid artery laceration with basilar subarachnoid hematoma. J Forensic Sci 2003;48(6):1366-8.

26. Rinkel GJ. Intracranial aneurysm screening: indications and advice for practice. Lancet Neuro. 2005;4(2):122-8. 